Revista Calidad en la Educación Superior

Programa de Autoevaluación Académica

Universidad Estatal a Distancia, Costa Rica

ISSN 1659-4703

revistacalidad@uned.ac.cr

\title{
PERFIL SOCIO DEMOGRÁFICO Y ACADÉMICO-CULTURAL DE LOS ESTUDIANTES DE NUEVO INGRESO A LA UNIVERSIDAD DE CIENCIAS MÉDICAS, PERIODO 2010-2014
}

\author{
DEMOGRAPHIC PROFILE AND SOCIO-CULTURAL ACADEMIC OF \\ UNIVERSITY OF MEDICAL SCIENCES NEW ADMISSIONS STUDENT, \\ PERIOD 2010-2014
}

\begin{abstract}
Hilda M. Sancho Ugalde ${ }^{1}$, hmsancho@yahoo.com Juan C. Vanegas Pissa ${ }^{2}$, pissacr2011@gmail.com Universidad de Ciencias Médicas (UCIMED), Costa Rica
\end{abstract}

Volumen 6, número 1

Mayo 2015

Pp. $1-18$

Recibido: 25 de mayo, 2014

Aprobado: 10 de marzo, 2015

${ }^{1}$ Hilda María Sancho Ugalde. Profesora emérita de la UCR. Doctora en Medicina y Cirugía. Maestría en Ciencias Biomédicas. Coordinadora de la Unidad de Aseguramiento de la Calidad Académica, Universidad de Ciencias Médicas y la Fisiología Humana. Correo electrónico: hmsancho@yahoo.com

2Juan Carlos Vanegas Pissa. Licenciado en Matemáticas y Física, Universidad de los Llanos. Egresado del programa de maestría en Bioestadística, Universidad de Chile. Maestría académica en Estadística, Universidad de Costa Rica. Estadístico de la Universidad de Ciencias Médicas. Consultor externo para el Estado de la Nación y de la Educación. Correo electrónico. pissacr2011@gmail.com 
Perfil socio demográfico y académico-cultural de los estudiantes de nuevo ingreso a la Universidad de Ciencias Médicas, periodo 2010-2014.

Hilda M. Sancho Ugalde y Juan C. Vanegas Pissa

\section{Resumen}

La Unidad de Aseguramiento de la Calidad de la Universidad de Ciencias Médicas (UCIMED) inició el programa de análisis de las características de la población estudiantil de nuevo ingreso, con el objetivo de proporcionar un insumo a las cátedras de la universidad para diseñar estrategias metodológicas conducentes a mejorar las habilidades requeridas para alcanzar el logro de los objetivos de formación, y prevenir las situaciones de abandono de los estudios. Mediante un formulario estructurándose recolectó información sobre el perfil sociodemográfico y se determinó el índice académico-cultural de las cohortes entre el 2010 y 2014. Los resultados indican las fortalezas y las debilidades estudiantiles y sirve de base para la gestión, en el ámbito académico, de los planes de estudios.

Palabras clave: perfil de estudiantes de nuevo ingreso, índice académico-cultural, abandono.

\section{Abstract}

The UCIMED Quality Assurance Unit began in 2010 the analysis of characteristics of the incoming student population in order to provide an input to the chair of the university to design leading methodological strategies to improve required skills to achieve the training objectives, and prevent situation of dropout. Using a structured formulary, the socio demography profile and academic-cultured index of cohorts between 2010 and 2014 were collected. The results indicate the strengths and weaknesses of the student population and provide bases of management of the academic curriculum.

Keywords: student profile, academic-cultural index, dropout.

La mayoría de las carreras del Área de la Salud, y la Medicina en particular, utilizan una serie de criterios para la selección de los alumnos de nuevo ingreso, que a su vez permiten correlacionarlos con el rendimiento universitario, las probabilidades de permanencia y el logro en sus estudios (Santelices, 2007).

Estos criterios que esencialmente se refieren a pruebas cognitivas y psicométricas, se han ampliado desde los trabajos de Bourdieu $(1970,1987)$, Cate (2002), Garay (2003), Hernández (2005) y Casillas (2007), al señalar que son las 
Perfil socio demográfico y académico-cultural de los estudiantes de nuevo ingreso a la Universidad de Ciencias Médicas, periodo 2010-2014.

Hilda M. Sancho Ugalde y Juan C. Vanegas Pissa

condiciones materiales y los recursos educativos familiares para el estudio, el nivel cultural y la capacidad de razonamiento que han adquirido de previo, las que juegan un papel crucial en el desempeño y posibilidades de obtener un buen resultado académico y éxito escolar de los jóvenes. Así el capital cultural se da en forma de hábitos de vida y conocimientos expertos o habilidades específicas que se adquieren e incorporan después de largos procesos de socialización, o sea al interior de la familia y en los estudios previos a su ingreso a la universidad.

Se tiene como objetivo analizar las características de la población estudiantil de nuevo ingreso en una universidad especializada en carreras de campo de la salud. Esto debido a que, por sus calidades y apoyo académico cultural familiar adquirido de previo, pueden influir en su rendimiento académico, que permitiría aproximar las probabilidades de tener éxito en su carrera.

A partir de la década de los sesenta, la demanda de estudios superiores se ha incrementado por parte de las poblaciones estudiantiles, con lo que la composición de la misma ha variado, diversificándose no solo en los aspectos demográficos y socioeconómicos sino también en la composición con el ingreso de las mujeres a la educación superior y la ampliación de la cobertura de estudiantes de secundaria.

El concepto de capital cultural fue operacionalizado mediante lo que se ha denominado "índice de apoyo académico-cultural", el cual comprende cinco subgrupos que pretende analizar la trayectoria cultural de la familia (bienes en el hogar, ocupación del padre y de la madre, escolaridad del padre y de la madre de familia).

Este índice representa el conjunto de recursos que posee el nuevo estudiante derivados de la influencia familiar tales como relaciones y contactos sociales, 
Perfil socio demográfico y académico-cultural de los estudiantes de nuevo ingreso a la Universidad de Ciencias Médicas, periodo 2010-2014.

Hilda M. Sancho Ugalde y Juan C. Vanegas Pissa

educación brindada en el hogar, recursos intelectuales y materiales, que cuenta de manera diferenciada la familia de origen, que distinguen a los individuos y expresan una historia social acumulada producto de la vida familiar. Aquellos que tienen más de estos recursos tienen mayor probabilidad de éxito en sus estudios y obtener beneficios ganados por sus propios méritos.

\section{Metodología}

\section{Tipo de estudio}

Es un estudio comparativo descriptivo y poblacional de los resultados obtenidos en las investigaciones realizadas en un periodo de cinco años (2010-2014).

Tabla 1. Población estudiada. Periodo 2010-2014.

\begin{tabular}{|l|c|c|c|c|c|c|c|c|}
\hline \multicolumn{1}{|c|}{ Año } & $\mathbf{2 0 1 0}$ & $\mathbf{2 0 1 1}$ & \multicolumn{2}{|c|}{2012} & \multicolumn{2}{|c|}{$\mathbf{2 0 1 3}$} & $\mathbf{2 0 1 4}$ & \multirow{2}{*}{ Totales } \\
\hline \multicolumn{1}{|c|}{ Semestre } & I & II & I & II & I & II & I & \\
\hline $\begin{array}{l}\text { Estudiantes } \\
\text { admitidos }\end{array}$ & 304 & 150 & 401 & 162 & 387 & 168 & 380 & $\mathbf{1 9 5 2}$ \\
\hline $\begin{array}{l}\text { Estudiantes } \\
\text { que } \\
\text { respondieron la } \\
\text { encuesta }\end{array}$ & 286 & 131 & 333 & 136 & 290 & 130 & 206 & $\mathbf{1 5 1 2}$ \\
$\begin{array}{l}\text { Porcentaje } \\
\text { Respuesta }\end{array}$ & 94.0 & 87.3 & 83.0 & 83.9 & 74.9 & 77.3 & 54.2 & $\mathbf{7 7 . 4}$ \\
\hline
\end{tabular}

Fuente: Elaboración propia, 2014

\section{Procedimiento}

Se utilizó un instrumento denominado "Perfil de ingreso" que consta de 78 ítems, distribuidos en aspectos demográficos, capital académico cultural y motivaciones para escoger la carrera, previamente validado y administrado durante el proceso de inducción al ingreso al primer semestre de la carrera escogida, durante el Taller de Técnicas de Estudio, con la ayuda del docente. 
Perfil socio demográfico y académico-cultural de los estudiantes de nuevo ingreso a la Universidad de Ciencias Médicas, periodo 2010-2014.

Hilda M. Sancho Ugalde y Juan C. Vanegas Pissa

Los datos se clasificaron en las siguientes categorías: características demográficas, características familiares, hábitos y metodologías de estudio, capital cultural, con sus dos componentes: familiar y escolar, índice de apoyo académicocultural, quese construyó como el promedio usual de los siguientes cinco índices estandarizados:

$>$ Índice de tenencia de bienes en el hogar (ITB)

$>$ Índice de ocupación del padre de familia (IOP)

$>$ Índice de ocupación de la madre de familia (IOM)

$>$ Índice de escolaridad del padre de familia (IEP)

$>$ Índice de escolaridad de la madre de familia (IEM)

Este índice de apoyo académico-cultural se agrupó en cuatro categorías de acuerdo a los cuartiles; Bajo, Medio bajo, Medio alto y Alto:

- Para el Análisis de los datos se utilizaron los estadísticos generales

- Para los índices se determinó el Coeficiente Alfa de Cronbach

\section{Análisis y discusión de resultados}

\section{Características demográficas generales:}

\section{Edad}

Al momento de ingreso, el promedio de edad de los estudiantes está entre 18 y 19 años, lo que indica que son estudiantes que acaban de concluir los estudios de secundaria (tabla 2).

Cabe destacar que el promedio de edad de ingreso es bajo para este tipo de carreras, en comparación con universidades de otras latitudes, debido a que se realiza inmediatamente después de haber aprobado los estudios de la secundaria. 
Perfil socio demográfico y académico-cultural de los estudiantes de nuevo ingreso a la Universidad de Ciencias Médicas, periodo 2010-2014.

Hilda M. Sancho Ugalde y Juan C. Vanegas Pissa

El ingreso al grado de licenciatura no contempla cursos o programas como requisito previo, tal como sucede en países anglosajones y europeos, que requieren estudios de nivel de bachillerato universitario o similar en ciencias, que demandan entre dos a tres años de estudios universitario previos al ingreso a la licenciatura o al doctorado en medicina, (MD). Esta situación da como resultado el tener una población estudiantil menor de 19 años al ingreso, con pocos alumnos con edades que superan los 30 años.

El proceso de admisión en la UCIMED comprende pruebas cognitivas, psicométricas y diferentes entrevistas con psicólogo, médico y autoridades, y se realiza inmediatamente después que el estudiante ha egresado de la secundaria esta situación incide en el tipo de población que se admite de primer ingreso, y pretende que la misma cumpla con el perfil de ingreso que las carreras del campo de la salud (Medicina, Farmacia, Microbiología, Fisioterapia y Nutrición ) requieren en cuanto a conocimientos, habilidades, destrezas y valores.

Tabla 2. Edad de ingreso a las carreras de salud en la UCIMED. Periodo 2010-2014

\begin{tabular}{|l|c|c|c|c|c|c|c|}
\hline \multicolumn{1}{|c|}{ Año } & 2010 & $\mathbf{2 0 1 1}$ & \multicolumn{2}{|c|}{2012} & \multicolumn{2}{|c|}{2013} & 2014 \\
\hline Semestre & I & II & I & II & I & II & I \\
\hline Más joven & 16 & 16 & 16 & 17 & 16 & 17 & 16 \\
\hline $\begin{array}{l}\text { Promedio de } \\
\text { edad }\end{array}$ & $\begin{array}{c}18.0 \pm 2 . \\
1\end{array}$ & $19.0 \pm 2.7$ & $17.9 \pm 2.0$ & $19.0 \pm 3.6$ & $18.2 \pm 3.0$ & $18.8 \pm 2.0$ & $18.1 \pm 2.0$ \\
\hline Más adulto & 36 & 35 & 34 & 40 & 41 & 30 & 33 \\
\hline
\end{tabular}

Fuente: Elaboración propia, 2014

\section{Sexo y estado civil}

En la tabla 3, se observa que el mayor porcentaje de la población que ingresa es femenina, con cifras que varían del $63.0 \%$ al $79.0 \%$ y solteras en ( $96.0 \%$ o más). 
Perfil socio demográfico y académico-cultural de los estudiantes de nuevo ingreso a la Universidad de Ciencias Médicas, periodo 2010-2014.

Hilda M. Sancho Ugalde y Juan C. Vanegas Pissa

El predominio femenino se ha venido haciendo más patente en el campo de la salud a nivel nacional, tanto en las instituciones estatales como en la mayoría de las privadas. Tendencia señalada por Boucourt (2006) en Venezuela, Panamá, Francia, México y España y por Javariz (2007) en Puerto Rico.

Este aumento en la población femenina en profesiones, que tradicionalmente habían sido un campo masculino, se debe entre otros aspectos, a que la oferta nacional de estas carreras es mayor, la mujer ha considerado que esas carreras le permiten realizarse desde el punto de vista vocacional, el deseo de servicio a los semejantes y además considera que tiene las aptitudes y capacidades intelectuales necesarias para tener éxito en carreras tan demandantes desde un punto de vista académico, así como que el mercado de trabajo les permite laborar en una profesión independiente, con expectativas de ingresos económicos elevados. Estas razones superan el $90 \%$ de las opiniones brindadas por los estudiantes en la colección de la información (tabla 3 y 10). También, se destaca que el mayor porcentaje son solteros, aspecto que permite una mayor dedicación al estudio, al no tener responsabilidades como las que demanda el establecimiento de una familia. 
Tabla 3. Distribución porcentual por sexo y estado civil. Periodo 2010-2014

\begin{tabular}{|c|c|c|c|c|c|c|c|}
\hline Año & $\mathbf{2 0 1 0}$ & $\mathbf{2 0 1 1}$ & \multicolumn{2}{|c|}{2012} & \multicolumn{2}{|c|}{2013} & $\mathbf{2 0 1 4}$ \\
\hline Semestre & I & II & I & II & I & II & I \\
\hline Femenino & 68.0 & 63.0 & 70.3 & 66.2 & 68.4 & 74.6 & 66.0 \\
\hline Masculino & 32.0 & 37.0 & 30.0 & 34.0 & 22.0 & 26.0 & 35.0 \\
\hline Solteros & 97.6 & 96.9 & 98.0 & 96.3 & 98.3 & 96.1 & 98.0 \\
\hline
\end{tabular}

Fuente: Elaboración propia.

\section{Características familiares}

Las familias son nucleares, pequeñas integradas por ambos padres y dos o tres hermanos (60.0-67.0\%) seguidos por el tipo uniparental. En los últimos dos años el grupo de estudiantes cuyo núcleo familiar está constituido únicamente por la madre ha venido aumentando, alcanzando un $23.9 \%$ en el 2014 y a partir del 2012se tienen estudiantes que conviven únicamente con su padre, el grupo que convive con otros familiares ha variado entre el $25.0 \%$ y el $42.0 \%$.

Más del 39.7\% de los estudiantes ocupan la posición de hijo mayor en familias en las cuales sus padres son profesionales (36.9.0-57.4\%) indicando que no es el primer individuo de esa familia que realiza estudios universitarios, lo cual le da un entorno cultural y académico, que le apoya a su formación. Además el residir en el seno de la familia le garantiza un entorno con facilidades para la realización de sus estudios y por tanto de permanencia en la carrera, característica que está en contraposición con los estudios de Boucourt \& González (2006), quienes hallaron que el $83.0 \%$ de los padres de su población estudiantil de nuevo ingreso en la Universidad de Pamplona, Colombia, tienen padres que no poseen título universitario y que es un factor crítico para explicar el abandono prematuro de estudios universitarios. 
Perfil socio demográfico y académico-cultural de los estudiantes de nuevo ingreso a la Universidad de Ciencias Médicas, periodo 2010-2014.

Hilda M. Sancho Ugalde y Juan C. Vanegas Pissa

Tabla 4. Características familiares. Período 2010-2014.

\begin{tabular}{|l|c|c|c|c|c|c|c|}
\hline \multicolumn{1}{|c|}{ Año } & $\mathbf{2 0 1 0}$ & $\mathbf{2 0 1 1}$ & \multicolumn{2}{|c|}{$\mathbf{2 0 1 2}$} & \multicolumn{2}{c|}{$\mathbf{2 0 1 3}$} & $\mathbf{2 0 1 4}$ \\
\hline \multicolumn{1}{|c|}{ Semestre } & $\mathbf{I}$ & $\mathbf{I I}$ & $\mathbf{I}$ & $\mathbf{I I}$ & $\mathbf{I}$ & $\mathbf{I I}$ & $\mathbf{I}$ \\
\hline $\begin{array}{l}\text { Convive con ambos } \\
\text { padres y hermanos }\end{array}$ & 60.1 & 55.7 & 67.9 & 64.0 & 68.3 & 61.5 & 67.8 \\
\hline $\begin{array}{l}\text { Convive con solo la } \\
\text { madre }\end{array}$ & 31.5 & 14.3 & 18.6 & 19.9 & 16.6 & 14.6 & 23.9 \\
\hline $\begin{array}{l}\text { Convive con solo el } \\
\text { padre }\end{array}$ & 0.0 & 0.0 & 2.1 & 4.4 & 2.1 & 2.3 & 7.3 \\
\hline $\begin{array}{l}\text { Convive con otros } \\
\text { familiares }\end{array}$ & 42.7 & 31.3 & 25.2 & 30.9 & 26.2 & 23.1 & 42.7 \\
\hline Es el hijo mayor & 43.0 & 41.0 & 43.2 & 50.4 & 39.7 & 49.1 & 42.9 \\
\hline Segundo hijo & 33.0 & 37.0 & 30.0 & 28.0 & 34.0 & 24.0 & 31.0 \\
\hline Es el tercer hijo & 18.0 & 16.0 & 16.0 & 11.0 & 16.0 & 18.0 & 17.0 \\
\hline Padres profesionales & 56.6 & 53.4 & 57.4 & 47.8 & 58.6 & 36.9 & 54.4 \\
\hline
\end{tabular}

Fuente: Elaboración propia, 2014

\section{Procedencia geográfica de los estudiantes}

Más del $70.0 \%$ de los estudiantes provienen de colegios situados en el Valle Central, de las provincias de San José, Alajuela y Heredia, o sea residen en zonas urbanas, muy pocos provienen de las provincias costeras de Limón, Puntarenas o Guanacaste. 
Perfil socio demográfico y académico-cultural de los estudiantes de nuevo ingreso a la Universidad de Ciencias Médicas, periodo 2010-2014.

Hilda M. Sancho Ugalde y Juan C. Vanegas Pissa

Tabla 5. Procedencia geográfica de los estudiantes, clasificación por provincia. Período 2010-2014

\begin{tabular}{|l|c|c|c|c|c|c|c|}
\hline \multicolumn{1}{|c|}{ Año } & $\mathbf{2 0 1 0}$ & $\mathbf{2 0 1 1}$ & \multicolumn{2}{|c|}{$\mathbf{2 0 1 2}$} & \multicolumn{2}{c|}{$\mathbf{2 0 1 3}$} & $\mathbf{2 0 1 4}$ \\
\hline Semestre & I & II & I & II & I & II & I \\
\hline San José & 42.0 & 53.0 & 37.0 & 45.0 & 43.0 & 43.0 & 44.0 \\
\hline Alajuela & 19.0 & 9.0 & 22.0 & 22.0 & 18.0 & 16.0 & 17.0 \\
\hline Heredia & 20.0 & 18.0 & 15.0 & 12.0 & 16.0 & 15.0 & 17.0 \\
\hline Cartago & 10.0 & 8.0 & 9.0 & 9.0 & 7.0 & 6.0 & 6.0 \\
\hline Guanacaste & 3.0 & 7.0 & 7.0 & 3.0 & 4.0 & 4.0 & 7.0 \\
\hline Limón & 3.0 & 3.0 & 3.0 & 4.0 & 5.0 & 2.0 & 4.0 \\
\hline Puntarenas & 3.0 & 2.0 & 7.0 & 5.0 & 7.0 & 2.0 & 5.0 \\
\hline Total & 100.0 & 100.0 & 100.0 & 100.0 & 100.0 & $88.0 *$ & 100.0 \\
\hline
\end{tabular}

Fuente: Elaboración propia, 2014

*En este semestre 12 personas no indicaron la provincia de procedencia.

Las distancias que deben recorrer para asistir a la universidad no son extensas y además pueden mantenerse residiendo con sus padres, situación que incide en los aspectos económicos y también en las posibilidades de acceso fácil a todos los recursos de apoyo académico que oferta la institución.

\section{Hábitos de estudio}

Las prácticas académicas más relevantes que esta población lleva a cabo para realizar sus estudios son: el estudio individual, las lecturas de libros de texto, la lectura de apuntes propios y las búsquedas en Internet, en porcentajes superiores al $80 \%$, y a su vez las que menos utilizan son el estudio en biblioteca y lectura de apuntes de compañeros. Dedican entre 2 a 11 horas semanales para realizar estudio independiente, lo cual traduce que, el hábito de estudio y la capacidad de esfuerzo sostenido deberá ser fortalecido porque es un factor que tiene un papel muy importante en la posibilidad de éxito de estas carreras, las cuales son muy 
Perfil socio demográfico y académico-cultural de los estudiantes de nuevo ingreso a la Universidad de Ciencias Médicas, periodo 2010-2014.

Hilda M. Sancho Ugalde y Juan C. Vanegas Pissa

demandantes, y en la mayoría de los casos, son de dedicación de tiempo completo y exclusivo.

Hernández (2005), señala que la permanencia en la Facultad de Medicina está fortalecida a través del orden, el hábito de estudio y la capacidad de esfuerzo sostenido que denota el estudiante. En este sentido la recomendación internacional es dedicar al menos dos horas diarias al estudio de temas pertinentes de la carrera; esto fuera de la asistencia a las actividades propias de la misma. Asimismo, el peso del crédito en los planes de estudios, usualmente supera los 20 créditos por periodo académico, lo cual indica una demanda mayor de tiempo de dedicación al estudio extraclase relacionado con los temas de los programas de curso. Todos esos aspectos inciden en las posibilidades de permanencia y éxito en la carrera. Los resultados evidenciados en este estudio, indican que el tiempo dedicado a desarrollo de las actividades de aprendizaje no es suficiente por cuanto dedican entre un $2.0 \%$ y $11.1 \%$ del total de horas semanales a estudio individual independiente. 
Perfil socio demográfico y académico-cultural de los estudiantes de nuevo ingreso a la Universidad de Ciencias Médicas, periodo 2010-2014.

Hilda M. Sancho Ugalde y Juan C. Vanegas Pissa

Tabla 6. Hábitos de estudio.Período 2010-2014.

\begin{tabular}{|l|c|c|c|c|c|c|c|}
\hline \multicolumn{1}{|c|}{ Año } & $\mathbf{2 0 1 0}$ & $\mathbf{2 0 1 1}$ & \multicolumn{2}{c|}{$\mathbf{2 0 1 2}$} & \multicolumn{2}{c|}{$\mathbf{2 0 1 3}$} & $\mathbf{2 0 1 4}$ \\
\hline \multicolumn{1}{|c|}{ Semestre } & I & II & I & II & I & II & I \\
\hline Estudio individual & 98.0 & 95.0 & 98.0 & 96.0 & 98.0 & 96.0 & 98.0 \\
\hline $\begin{array}{l}\text { Lectura de libro } \\
\text { de texto }\end{array}$ & 96.0 & 95.0 & 95.0 & 92.0 & 96.0 & 92.0 & 92.0 \\
\hline $\begin{array}{l}\text { Lectura de } \\
\text { apuntes propios }\end{array}$ & 96.0 & 98.0 & 96.0 & 96.0 & 96.0 & 98.0 & 98.0 \\
\hline $\begin{array}{l}\text { Búsqueda en } \\
\text { internet }\end{array}$ & 89.0 & 88.0 & 84.0 & 92.0 & 88.0 & 88.0 & 88.0 \\
\hline $\begin{array}{l}\text { Estudio en } \\
\text { biblioteca }\end{array}$ & 44.0 & 58.0 & 42.0 & 28.0 & 52.0 & 59.0 & 56.0 \\
\hline $\begin{array}{l}\text { Horas estudio } \\
\text { individual } \\
\text { independiente } \\
\text { semanal }\end{array}$ & $4.6 \mathrm{a}$ & $2.0 \mathrm{a}$ & $3.2 \mathrm{a}$ & $2.5 \mathrm{a}$ & $3.2 \mathrm{a}$ & $2.5 \mathrm{a}$ & $3.1 \mathrm{a}$ \\
7.2 & 8.0 & 7.6 & 7.5 & 10.6 & 5.8 & 11.1 \\
\hline
\end{tabular}

Fuente: Elaboración propia, 2014

Nota: esta variable es de respuesta múltiple

\section{Colegio de procedencia y financiamiento de estudios}

La mayoría de la población proviene de colegios privados, sin embargo este porcentaje ha variado, de ser más del $76.4 \%$ en el 2010 , a un $67.3 \%$ en el 2014 . Con un incremento de los alumnos provenientes de colegios públicos, pasando del $11,7 \%$ al $44,4 \%$ en el 2013 ,y con un $25.0 \%$ en el 2014 . El grupo proveniente de colegios subvencionados se ha mantenido bastante estable, entre el $7.0 \%$ y el $10.0 \%$ (tabla 7 ).

El financiamiento de sus estudios universitarios prioritariamente lo harán a través del apoyo económico de sus padres, algunos de ellos tendrán apoyo mixto por parte de los padres, y por parte del Consejo Nacional de Préstamos para la Educación Superior (CONAPE). Es una población estudiantil que proviene de 
Perfil socio demográfico y académico-cultural de los estudiantes de nuevo ingreso a la Universidad de Ciencias Médicas, periodo 2010-2014.

Hilda M. Sancho Ugalde y Juan C. Vanegas Pissa

familias que en más del $70 \%$ cuentan con casa propia, y tienen condiciones económicas que les permiten financiar los estudios, e apoyo económico de los padres para el pago de la matrícula y gastos relacionados con el estudio es muy importante, y traduce una población estudiantil que no trabaja. (tabla 8).

Tabla 7. Colegio de procedencia. Período 2010-2014

\begin{tabular}{|l|c|c|c|c|c|c|c|}
\hline \multicolumn{1}{|c|}{ Porcentajes } \\
\hline \multicolumn{1}{|c|}{ Año } & $\mathbf{2 0 1 0}$ & $\mathbf{2 0 1 1}$ & \multicolumn{2}{|c|}{$\mathbf{2 0 1 2}$} & \multicolumn{2}{|c|}{$\mathbf{2 0 1 3}$} & $\mathbf{2 0 1 4}$ \\
\hline \multicolumn{1}{|c|}{ Semestre } & I & II & I & II & I & II & I \\
\hline Privado & 76.4 & 65.2 & 64.8 & 51.9 & 50.6 & 46.7 & 67.3 \\
& $(13)$ & $(15)$ & $(46)$ & $(42)$ & $(78)$ & $(42)$ & $(37)$ \\
\hline Público & 11.7 & 21.7 & 25.3 & 39.2 & 40.4 & 44.4 & $25(13)$ \\
& $(2)$ & $(5)$ & $(18)$ & $(30)$ & $(64)$ & $(40)$ & \\
\hline Subvencionado & 11.7 & 13.04 & $9.8(7)$ & $8.9(7)$ & 9.0 & $8.8(8)$ & $7.6(4)$ \\
& $(2)$ & $(3)$ & & & $(14)$ & & \\
\hline Total & 100.0 & 100.0 & 100.0 & 100.0 & 100.0 & 100.0 & 100.0 \\
& $(17)$ & $(23)$ & $(71)$ & $(79)$ & $(156)$ & $(90)$ & $(54)$ \\
\hline
\end{tabular}

Fuente: Elaboración propia, 2014

Tabla 8. Financiamiento de sus estudios.Período 2010-2013

\begin{tabular}{|l|c|c|c|c|c|c|}
\hline \multicolumn{1}{|c|}{ Año } & $\mathbf{2 0 1 0}$ & $\mathbf{2 0 1 1}$ & \multicolumn{2}{|c|}{$\mathbf{2 0 1 2}$} & \multicolumn{2}{c|}{$\mathbf{2 0 1 3}$} \\
\hline \multicolumn{1}{|c|}{ Semestre } & I & II & I & II & I & II \\
\hline $\begin{array}{l}\text { Financiamiento } \\
\text { padres }\end{array}$ & 98.9 & 96.0 & 98.0 & 91.1 & 91.0 & 93.0 \\
\hline Préstamo CONAPE & 18.9 & 36.4 & 13.0 & 4.7 & 9.0 & 17.0 \\
\hline
\end{tabular}

Fuente: Elaboración propia, 2014

Índice de apoyo académico-cultural

En la tabla 9 se muestran los resultados. La población estudiantil proviene en porcentajes superiores al $50.0 \%$ de padres profesionales con estudios universitarios de nivel de grado y en algunos postgrado. Esta característica, sumada a que la mayoría son el hijo mayor, les permite tener mayores referentes 
Perfil socio demográfico y académico-cultural de los estudiantes de nuevo ingreso a la Universidad de Ciencias Médicas, periodo 2010-2014.

Hilda M. Sancho Ugalde y Juan C. Vanegas Pissa

y aportes académicos de su propia familia, debido a que mucho de su trabajo estudiantil lo realizan al interior del hogar, donde tienen textos y tecnología que les permite realizar los mismos en condiciones óptimas. Dichas condiciones benefician la actividad de aprendizaje porque su propia familia es un interlocutor que al poseer un nivel cultural académico alto, permite una interacción provechosa para el estudiante.

En esta población el promedio del índice de apoyo académico es de $0.66 \pm 0.24$, donde uno es el máximo y cero el mínimo; asimismo se localizan entre apoyo medio alto y alto para valores de la población en estudio entre el $60.0 \%$ y $83.0 \%$. Los valores de consistencia interna de este indicador oscilan en los periodos en estudio entre buenos y excelentes.

Es una población estudiantil que cuenta con apoyo académico y cultural que permite esperar buenos resultados académicos en el desarrollo de su carrera.

Tabla 9. Índice de apoyo académico cultural. Período 2010-2014

\begin{tabular}{|c|c|c|c|c|c|c|c|}
\hline \multicolumn{8}{|c|}{ Porcentajes } \\
\hline Año & 2010 & 2011 & \multicolumn{2}{|c|}{2012} & \multicolumn{2}{|c|}{2013} & 2014 \\
\hline Semestre & 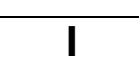 & II & I & II & $\mathrm{I}$ & II & I \\
\hline $\begin{array}{l}\text { IAAC } \\
\text { promedio }\end{array}$ & $\begin{array}{c}0.67 \pm \\
0.23\end{array}$ & $\begin{array}{c}0.64 \pm \\
0.25\end{array}$ & $\begin{array}{c}0.64 \pm \\
0.25\end{array}$ & $\begin{array}{c}0.65 \pm \\
0.25\end{array}$ & $\begin{array}{c}0.70 \pm \\
0.24\end{array}$ & $\begin{array}{c}0.59 \pm \\
0.26\end{array}$ & $\begin{array}{c}0.71 \pm \\
0.21\end{array}$ \\
\hline $\begin{array}{l}\text { Medio- } \\
\text { Alto }\end{array}$ & 70.6 & 68.7 & 47.3 & 66.2 & 82.0 & 60.6 & 82.9 \\
\hline $\begin{array}{l}\text { Medio } \\
\text { bajo } \\
\text { bajo }\end{array}$ & 29.4 & 31.3 & 52.6 & 33.8 & 18.0 & 39.4 & 17.0 \\
\hline $\begin{array}{l}\text { Alfa de } \\
\text { Cronbach }\end{array}$ & 0.9 & 0.9 & 0.8 & 0.8 & 0.8 & 0.8 & 0.8 \\
\hline
\end{tabular}

Fuente: Elaboración propia, 2014 
Perfil socio demográfico y académico-cultural de los estudiantes de nuevo ingreso a la Universidad de Ciencias Médicas, periodo 2010-2014.

Hilda M. Sancho Ugalde y Juan C. Vanegas Pissa

\section{Razones para escoger la carrera}

La razón por la que los estudiantes eligen una carrera se considera como uno de los elementos que más influencia el desempeño académico en el desarrollo de la misma.

En la población en estudio las razones por las que desea estudiar la carrera a la que se inscribió son principalmente por gusto personal, por ayudar a la gente, llegar a ser profesional independiente y considerar que tienen las habilidades para la profesión escogida, con porcentajes entre el $70.0 \%$ y el $100.0 \%$. En porcentajes menores al $50 \%$ se localizan otras motivaciones, tales como la presión familiar o de sus, amigos, otros profesionales o la existencia de una empresa familiar.

En Costa Rica, en el campo de la salud, esta decisión es probable que esté influenciada por el propio sistema de salud, el cual ha demostrado ser permanente y sólido y de alta calidad. Al ser un sistema declarado constitucionalmente como universal, cubre a toda la población y, por lo tanto, requiere de los recursos humanos necesarios para cumplir con ese mandato, por ello, la posibilidad de empleo a futuro al interior del mismo y ser un profesional independiente podría estar como fundamento de la decisión.

Así muchos jóvenes tienen interés en llegar a ser parte de dicha institución, motivación que se refuerza cuando sus padres son funcionarios de la misma. 
Perfil socio demográfico y académico-cultural de los estudiantes de nuevo ingreso a la Universidad de Ciencias Médicas, periodo 2010-2014.

Hilda M. Sancho Ugalde y Juan C. Vanegas Pissa

Tabla 10. Razones para estudiar ciencias de la salud. Período 2010-201

\begin{tabular}{|c|c|c|c|c|c|c|c|}
\hline \multicolumn{8}{|c|}{ Porcentajes } \\
\hline AÑO & 2010 & 2011 & & & & & 2014 \\
\hline SEMESTRE & I & II & I & II & I & II & I \\
\hline Gusto personal & 100 & 98 & 100 & 98 & 100 & 98 & 100 \\
\hline $\begin{array}{l}\text { Deseos de ayudar a } \\
\text { los semejantes }\end{array}$ & 100 & 96 & 98 & 96 & 98 & 98 & 98 \\
\hline $\begin{array}{l}\text { Ser profesional } \\
\text { independiente }\end{array}$ & 80 & 75 & 76 & 80 & 82 & 74 & 68 \\
\hline $\begin{array}{l}\text { Tener las } \\
\text { habilidades para la } \\
\text { profesión escogida }\end{array}$ & 92 & 78 & 88 & 86 & 86 & 78 & 90 \\
\hline Otras ${ }^{*}$ & $<50$ & $<50$ & $<50$ & $<50$ & $<50$ & $<50$ & \\
\hline
\end{tabular}

Fuente: Elaboración propia, 2014

Nota: esta variable es de respuesta múltiple.

\section{Conclusiones}

El estudio muestra que el grupo de estudiantes que ingresa a las carreras ofertadas por la UCIMED se caracteriza por ser una población:

1. Predominantemente femenina, soltera, menores de 19 años, primer hijo de la familia, la cual es nuclear, de padres profesionales universitarios, provenientes de las provincias del Valle Central y de colegios privados.

2. No trabajan y el financiamiento de los estudios es predominantemente a través del apoyo familiar.

3. Poseen un índice académico- cultural de medio alto a alto, lo que les dota de un capital cultural que les sirve de apoyo para enfrentar estudios de alta demanda.

4. Poseen débiles hábitos de estudio que deben ser mejorados y desarrollar habilidades y destrezas para enfrentar estudios de dedicación exclusiva y alta demanda de tiempo, esfuerzo y responsabilidad. 
Perfil socio demográfico y académico-cultural de los estudiantes de nuevo ingreso a la Universidad de Ciencias Médicas, periodo 2010-2014.

Hilda M. Sancho Ugalde y Juan C. Vanegas Pissa

5. La escogencia de la carrera la realizan esencialmente por motivaciones altruistas y deseos de ser profesionales independientes.

6. Ante esta tipo de población, se deben impulsar medidas que permitan desarrollar las actitudes necesarias para el estudio, el trabajo en equipo y la adquisición de hábitos de estudio, de acuerdo con las demandas que actualmente requieren los estudios en el campo de la salud de un nivel universitario, y a la vez prevenir el abandono de los mismos.

\section{Referencias}

Boucourt, J. \& González, M. (2006). Perfil socioeconómico y demográfico del estudiante de nuevo ingreso a la Universidad del Zulia. Análisis comparativo cohortes 98-99; 99-2000; 2000-2001; 2001-2002. Revista Venezolana de Ciencias Sociales, 10(1), 86-105.

Bourdieu, P. (1987). Los tres estados del capital cultural. En revista Sociológica, 5, $11-17$.

Cancino, S., Olivert, O., Peña, J., \& Velasco, A. (2012). Condiciones socioeconómicas del estudiante de pregrado de la universidad de Pamplona. Recuperado de http://www.unipamplona.edu.co/unipamplona/portallG/home_39/recursos/01 _general/18012013/caractsocioeconomico.pdf

Casillas, M. A., Chain, R., \& Jácome, N. (2007). Origen social de los estudiantes y trayectorias estudiantiles en la Universidad Veracruzana. Revista de la Educación Superior, XXXVI (2)(142), 7-29.

Cate, T \&\& Smal, K. (2002). Educational assessment center techniques entrance selection in medical school. Academic Medicine, 77, 737-739.

Garay, A. (noviembre-diciembre2003). El perfil de los estudiantes de nuevo ingreso de las universidades tecnológicas en México. El Cotidiano, 122, 7585.

Hernández, J. M., Hernández, R., Nieto, A. \& Hernández, J. F. (2005). Factores de riesgo para la deserción de estudiantes en la Facultad de Medicina de la Universidad Autónoma de San Luis Potosí (UASLP), México. Gaceta Médica de México, 141(5), 445-447.citado por Brito-Orta, M. D., Grimaldo- 
Perfil socio demográfico y académico-cultural de los estudiantes de nuevo ingreso a la Universidad de Ciencias Médicas, periodo 2010-2014.

Hilda M. Sancho Ugalde y Juan C. Vanegas Pissa

Avilé., J. I. \& Moreno-Tapia, J. A. (2010). Comparación de las trayectorias escolares como complemento en la evaluación de los cambios curriculares. GacMédMéx, 146(4), 2010. Recuperado de http://www.scielo.org.mx/scielo.php?script=sci_arttext\&pid=S0016$38132005000500016 \&$ Ing=es\&tlng=es

Javariz, G. (2007). Perfil de estudiantes de nuevo ingreso 2006-2007, Universidad de Puerto Rico en Aguadilla. Recuperado de http://www.uprag.edu/uploads/opei/Perfildeestudiantesdenuevoingreso2006 2007.pdf

Santelices, M.V. (2007). Procesos de admisión a instituciones de educación superior en el mundo: antecedentes bibliográficos para la consideración de criterios complementarios en el proceso de admisión a la Pontificia Universidad Católica de Chile. Recuperado de http://www7.uc.cl/webpuc/piloto/pdf/evidencias1.pdf 